\title{
Perfil das internações por Esclerose Múltipla entre residentes do município de Praia Grande (SP)
}

\author{
Profile of admissions for Multiple Sclerosis among residents \\ of the city of Praia Grande (SP)
}
Perfil de admisiones por Esclerosis Múltiple entre residentes de la ciudad de Praia Grande (SP)

\author{
Ana Paula Silva1, Rafaela de Melo Ventura1, \\ Maria do Socorro Morais Pereira Simões²
}

1.Acadêmica de Fisioterapia. Curso de Fisioterapia, Faculdade do Litoral Sul Paulista - FALS, Praia GrandeSP, Brasil.

2.Fisioterapeuta, Doutoranda pelo Programa de Ciências do Movimento Humano e Reabilitação, Departamento de Ciências do Movimento Humano, Universidade Federal de São Paulo - UNIFESP, SantosSP. Docente do Curso de Fisioterapia da Faculdade do Litoral Sul Paulista - FALS, Praia Grande-SP, Brasil.

\begin{abstract}
Resumo
Introdução. A Esclerose Múltipla (EM) consiste em uma doença inflamatória, crônica, degenerativa e autoimune que afeta diretamente a bainha de mielina do Sistema Nervoso Central. As pessoas com EM podem passar por períodos de exacerbações e remissões dos sintomas e, dependendo do tipo de complicação, é necessária a hospitalização. Objetivo. Analisar o perfil das internações por EM entre munícipes de Praia Grande (SP) no período de 10 anos. Método. Trata-se de um estudo observacional descritivo que utilizou dados públicos obtidos do Sistema de Informações de Saúde - DATASUS. Os dados foram analisados por meio de estatística descritiva. Resultados. Foram registradas 57 internações decorrentes da EM entre munícipes de Praia Grande no período estudado, com maior número de internações nos anos de 2018 e 2019. Houve predominância de indivíduos do sexo masculino, na faixa etária de 30 a 34 anos, da raça/cor branca, com tempo médio ajustado de internação de 8,9 dias. Todas as internações ocorreram em municípios diferentes do de residência do usuário. Conclusão. Sugerimos a elaboração de políticas públicas municipais e regionais para atender às demandas das pessoas com EM, de modo que o atendimento em todos os níveis de atenção possa ser dispensado o mais próximo possível da residência dos usuários.
\end{abstract}

Unitermos. Epidemiologia; Esclerose múltipla; Hospitalização

\begin{abstract}
Introduction. Multiple Sclerosis (MS) is an inflammatory, chronic, degenerative, and autoimmune disease that occurs directly with the myelin sheath of the Central Nervous System. People with MS may experience periods of symptom exacerbations and remissions and, depending on the type of complication, hospitalization is required. Objective. To analyze the profile of hospitalizations for MS among residents of Praia Grande (SP) over a 10-year period. Method. This is a descriptive observational study that uses public data, collected from the Health Information System - DATASUS. Data were analyzed using descriptive statistics. Results. There were 57 hospitalizations due to MS among residents of Praia Grande during the study period, with the highest number of hospitalizations in the years 2018 and 2019. There was a predominance of males, aged 30 to 34 years, of race/color white, with a mean time of hospital stay of 8.9 days. All admissions occurred in different cities from user's residence. Conclusion. We suggest the publication of municipal and regional public policies to meet the demands of people with MS, so that care at all levels of care can be provided as close as possible to the users' home.
\end{abstract}

Keywords. Epidemiology; Hospitalization; Multiple sclerosis 


\section{Resumen}

Introducción. La esclerosis múltiple $(E M)$ es una enfermedad inflamatoria, crónica, degenerativa y autoinmune que ocurre directamente con la vaina de mielina del Sistema Nervioso Central. Las personas con EM pueden experimentar períodos de exacerbaciones y remisiones de los síntomas y, según el tipo de complicación, se requiere hospitalización. Objetivo. Analizar el perfil de las hospitalizaciones por EM entre los residentes de Praia Grande (SP) durante un período de 10 años. Método. Se trata de un estudio observacional descriptivo que utiliza datos públicos recopilados del Sistema de Información en Salud - DATASUS. Los datos se utilizaron mediante estadística descriptiva. Resultados. Hubo 57 hospitalizaciones por EM entre los residentes de Praia Grande en el período estudiado, con el mayor número de hospitalizaciones en los años 2018 y 2019 . Hubo un predominio del sexo masculino, de 30 a 34 años, de raza/color blanco, con un tiempo medio de estancia hospitalaria de 8,9 días. Todas las admisiones se realizaron en los diferentes municipios donde reside el usuario. Conclusión. Sugerimos la publicación de políticas públicas municipales y autonómicas para atender las demandas de las personas con EM, para que la atención en todos los niveles de atención se pueda brindar lo más cerca posible del domicilio de los usuarios.

Palabras clave. Epidemiología; Esclerosis múltiple; Hospitalización

Trabalho realizado na Faculdade do Litoral Sul Paulista - FALS, Praia Grande-SP, Brasil.

\section{INTRODUÇÃO}

A Esclerose Múltipla (EM) consiste em uma doença inflamatória, crônica, degenerativa e autoimune que afeta diretamente a bainha de mielina do Sistema Nervoso Central. É considerada não só uma doença inflamatória como também neurodegenerativa ${ }^{1}$, provocando lesões medulares e cerebrais. Apesar de ter etiologia desconhecida, estudos apontam para fatores ambientais e genéticos envolvidos na fisiopatologia da $\mathrm{EM}^{2}$.

É uma doença que frequentemente se manifesta em jovens adultos com idade de 20 a 40 anos, sendo o sexo feminino o mais acometido ${ }^{3}$. As manifestações iniciais mais frequentes consistem na fraqueza muscular, fadiga, alterações visuais, piramidais, cerebelares, sensoriais, 
tronco encefálicas e esfincterianas ${ }^{4}$. As pessoas com EM podem passar por períodos de exacerbações e remissões dos sintomas e, dependendo do tipo de complicação, é necessária a hospitalização ${ }^{5}$.

Criado em 1991 a partir da instituição da Fundação Nacional de Saúde por meio do Decreto no 100, o DATASUS consiste em um Departamento de Informática do SUS responsável por fornecer informações e suporte de informática necessários ao processo de planejamento, operação e controle ${ }^{6}$. Além disso, o DATASUS fornece soluções para as Secretarias de Saúde Estaduais e Municipais, de acordo com as necessidades apontadas pelos respectivos gestores ${ }^{7}$. O DATASUS disponibiliza acesso amplo, irrestrito e gratuito a informações de saúde, morbidade, mortalidade e acesso a serviços, entre outras.

Considerando que uma das diretrizes do Sistema Único de Saúde (SUS) é planejar ações conforme as características epidemiológicas da população, é importante que se obtenha informações sobre os munícipes da Praia Grande com Esclerose Múltipla que precisaram de internação nos últimos anos. Essa informação seria de suma importância para auxiliar no planejamento estratégico de ações para acompanhamento desse perfil de pacientes.

Portanto, o objetivo deste trabalho foi analisar o perfil das internações por Esclerose Múltipla entre residentes do município de Praia Grande (SP) no período de outubro de 2010 a outubro de 2020. 


\section{MÉTODO}

Este é um estudo observacional descritivo, que utilizou dados obtidos do Sistema de Informações de Saúde (DATASUS). Considerando que foram analisados dados de domínio público, o presente estudo não foi submetido para apreciação por Comitê de Ética em Pesquisa.

Para o presente estudo, foram coletados dados sobre internação hospitalar por Esclerose Múltipla no município de Praia Grande (São Paulo), no período de outubro de 2010 a outubro de 2020. A busca na base de dados foi realizada em 9, 10 e 19 de janeiro de 2021 a partir da seção Epidemiológicas e Morbidade, grupo Morbidade Hospitalar do SUS (SIH/SUS), opção Geral por local de residência - a partir de 2008, e selecionamos o Estado de São Paulo.

Utilizando a ferramenta TABNET selecionamos a opção Município (Linha), Coluna não ativa e, em Conteúdo, selecionamos as opções internações, valor dos serviços hospitalares, valor médio de internação, dias de permanência e média de permanência hospitalar. Em Períodos disponíveis, selecionamos as opções de outubro de 2010 a outubro de 2020. A seguir, delimitamos as seguintes opções: Município (Praia Grande), Lista morbidades CID-10 (Esclerose múltipla), Faixa etária (todas as faixas etárias de 20 a 59 anos), Sexo (todas as categorias), e Cor/raça (todas as categorias). Em seguida repetimos a busca com as mesmas informações, substituindo o Município por Estabelecimento (Linha). 


\section{Análise Estatística}

Os dados foram analisados por meio de estatística descritiva, utilizando-se média e desvio padrão para variáveis contínuas, e número absoluto e proporção para variáveis categóricas.

\section{RESULTADOS}

No período analisado, de outubro de 2010 a outubro de 2020, foram registradas 57 internações decorrentes da EM entre munícipes de Praia Grande. O primeiro registro ocorreu no ano de 2011 e o maior número de internações foi nos anos de 2018 e 2019, com redução no ano seguinte.

Houve predominância de indivíduos do sexo masculino, na faixa etária de 30 a 34 anos, da raça/cor branca. As características da população estão apresentadas na Tabela 1.

O tempo de permanência hospitalar variou durante o período de análise, com aumento nos anos de 2015 e 2019. A média geral no período foi de 1,6 dia, com custo médio de $\mathrm{R} \$ 787,04$ por internação. As informações sobre o tempo e custo das internações estão apresentadas na Tabela 2.

A segunda etapa da busca, por estabelecimento de saúde, retornou que das 57 internações de residentes da Praia Grande, 9 ocorreram na cidade de Santos e 48 na cidade de São Paulo. As internações ocorreram em dois hospitais de cada cidade, sendo um hospital com 7 internações (estabelecimento A) e outro com 2 internações (estabelecimento B) em Santos, e um hospital com uma 


\section{internação (estabelecimento C) e outro com 47 internações (estabelecimento D) em São Paulo.}

Tabela 1. Características da população residente de Praia Grande, registrada com internações por Esclerose Múltipla no período de outubro de 2010 a outubro de 2020.

\begin{tabular}{cc}
\hline \multicolumn{1}{c}{ Variáveis } & N (\%) \\
\hline Sexo & \\
Masculino & $51(89,47)$ \\
Feminino & $6(10,53)$ \\
\hline Faixa etária & \\
20 a 24 anos & 0 \\
25 a 29 anos & $18(31,58)$ \\
30 a 34 anos & $30(52,63)$ \\
35 a 39 anos & $1(1,75)$ \\
40 a 44 anos & $4(7,02)$ \\
45 a 49 anos & $4(7,02)$ \\
50 a 54 anos & 0 \\
55 a 59 anos & 0 \\
\hline Cor/ raça & \\
Branco & $50(87,72)$ \\
Pardo & $5(8,77)$ \\
Sem informação & $2(3,51)$ \\
Preto, Amarelo e Indígena & 0 \\
\hline
\end{tabular}

$\mathrm{N}$ : número de indivíduos.

Tabela 2. Informações sobre o tempo de permanência e custo médio das internações por Esclerose Múltipla entre munícipes de Praia Grande no período de outubro de 2010 a outubro de 2020.

\begin{tabular}{rcccc}
\hline Ano & $\begin{array}{c}\text { Tempo de } \\
\text { permanência, dias }\end{array}$ & $\begin{array}{c}\text { Tempo médio de } \\
\text { permanência, dias }\end{array}$ & Custo médio, R\$ & Custo total, R\$ \\
\hline 2010 & 0 & 0 & 0 & 0 \\
2011 & 7 & 7,0 & 303,80 & 303,80 \\
2012 & 5 & 5,0 & 572,55 & 572,55 \\
2013 & 5 & 5,0 & 316,50 & 316,50 \\
2014 & 0 & 0 & 0 & 0 \\
2015 & 28 & 14,0 & 536,47 & $1.702,94$ \\
2016 & 0 & 0 & 303,80 & $3.038,00$ \\
2017 & 0 & 0 & 303,80 & $2.734,20$ \\
2018 & 7 & 0,6 & 311,92 & $3.743,04$ \\
2019 & 25 & 2,1 & $1.749,07$ & $20.988,84$ \\
2020 & 12 & 1,3 & $1.343,49$ & $12.091,43$ \\
\hline
\end{tabular}

R\$: real. 
$\mathrm{Na}$ Tabela 3 apresentamos as informações sobre permanência hospitalar por ano segundo os estabelecimentos de saúde. Nota-se que não há informações sobre o tempo de permanência no estabelecimento $D$, de São Paulo. Sendo assim, recalculamos o tempo médio de permanência sem considerar esse estabelecimento e constatamos que foi de 8,9 dias, apresentado na Tabela 4.

Tabela 3. Informações sobre o tempo de permanência das internações por Esclerose Múltipla entre munícipes de Praia Grande no período de outubro de 2010 a outubro de 2020, segundo os estabelecimentos de saúde.

Estabelecimento

Número de internações
Permanência total, dias

Média de

permanência, dias

\begin{tabular}{cccc}
\hline A & 7 & 64 & 9,1 \\
B & 2 & 20 & 10 \\
C & 1 & 5 & 5 \\
D & 47 & 0 & 0
\end{tabular}

Estabelecimentos A e B localizados na cidade de Santos, e estabelecimentos C e D localizados na cidade de São Paulo.

Tabela 4. Informações sobre o tempo de permanência das internações por Esclerose Múltipla entre munícipes de Praia Grande no período de outubro de 2010 a outubro de 2020 desconsiderando o estabelecimento D.

\begin{tabular}{lcc}
\hline Ano & Tempo de permanência, dias & $\begin{array}{c}\text { Tempo médio de } \\
\text { permanência, dias }\end{array}$ \\
\hline 2010 & 0 & 0 \\
2011 & 7 & 7 \\
2012 & 5 & 5 \\
2013 & 5 & 5 \\
2014 & 0 & 0 \\
2015 & 28 & 14 \\
2016 & 0 & 0 \\
2017 & 0 & 0 \\
2018 & 7 & 3,5 \\
2019 & 25 & 12,5 \\
2020 & 12 & 12 \\
Total & 89 & 8,9 \\
\hline
\end{tabular}




\section{DISCUSSÃO}

O objetivo deste trabalho foi analisar o perfil das internações por Esclerose Múltipla entre residentes do município de Praia Grande no período de outubro de 2010 a outubro de 2020. Observamos que o tempo médio ajustado de internação foi de 8,9 dias e que ocorreram em municípios diferentes do qual os pacientes residiam.

Diferentemente do que foi apresentado em outros estudos $^{8,9}$, houve predominância de indivíduos do sexo masculino internados por EM. Embora a doença seja mais prevalente entre pessoas do sexo feminino, a gravidade da neurodegeneração parece ser maior no sexo masculino ${ }^{10,11}$. Ainda assim, apesar da diferença no curso e gravidade da doença relatada entre os sexos, a maior prevalência de munícipes do sexo masculino que necessitaram de internação por EM merece investigação.

Atualmente estão descritas quatro formas de evolução clínica da EM: remitente-recorrente (EM-RR), primariamente progressiva (EM-PP), primariamente progressiva com surto (EM-PP com surto) e secundariamente progressiva (EM$\mathrm{SP})^{12}$. A mais comum, que representa aproximadamente $85 \%$ dos diagnósticos iniciais, é a EM-RR, caracterizada por surtos que duram semanas ou dias e depois desaparecem, podendo ter sua recuperação completa ou sequelas permanentes ${ }^{12}$. As formas EM-PP e EM-PP com surto perfazem de $10 \%$ a $15 \%$ de todos os casos, nas quais se descreve a ocorrência de surtos e declínios neurológicos ${ }^{13}$. 
Embora não tenhamos informações sobre qual é a forma de EM diagnosticada entre os munícipes de Praia Grande pois o DATASUS não fornece essa informação, é possível que seja do tipo EM-PP, apresentado em outro estudo como sendo a de maior prevalência no sexo masculino ${ }^{14}$.

O maior acometimento na faixa etária de 30 a 34 anos de raça/cor branca está em concordância com os achados de outros estudos, embora tenha sido observado maior distribuição de faixa etária, variando de 20 a 40 anos $^{15}$. A EM é geralmente diagnosticada na idade adulta jovem, quando os indivíduos geralmente estão estruturando suas carreiras ou constituindo família. A EM pode, portanto, impactar negativamente o bem-estar econômico e social das pessoas, bem como afetar seus familiares e parceiros ${ }^{12}$.

Das 57 internações registradas no período estudado, 9 ocorreram na cidade de Santos e 48 na cidade de São Paulo. Esse fato pode estar relacionado à própria organização do SUS em redes de atenção, pois não há centros de referência em EM no município de Praia Grande.

Embora o tempo médio de internação tenha sido de 1,6 dia, apuramos que um dos serviços de saúde não fornecia informações referentes à internação. Quando ajustamos considerando apenas os serviços com informações completas, o tempo médio de internação passou a ser de 8,9 dias. O sistema do DATASUS depende de informações fornecidas pelos serviços de saúde, e a falta dessas informações afeta diretamente a interpretação desses dados 
e as condições de morbidades previstas. Consequentemente, o planejamento das ações e serviços de saúde a partir de informações epidemiológicas pode ser afetado negativamente.

Ressaltamos também a importância do acompanhamento desses indivíduos pela Atenção Básica. Além do acompanhamento longitudinal de saúde, o acompanhamento desses pacientes após alta se faz necessário devido ao tempo de internação prolongado, com riscos de perda de força muscular e perdas funcionais ${ }^{16}$, além do risco para readmissão hospitalar ${ }^{17}$.

Embora não fosse possível extrair informações sobre a causa das internações, as principais nessa população estão relacionadas à piora dos sintomas ${ }^{5,17}$. Permanecendo internadas em média 8,9 dias, as internações podem afetar negativamente o convívio social e a qualidade de vida dos indivíduos com EM, além de gerar um custo médio de $\mathrm{R} \$ 3.058,27$ por internação. Considerando o tempo de internação maior do que o reportado ${ }^{5}$, de 3 a 5 dias, e o maior risco para readmissão hospitalar associado a tempos de internação de 7 a 9 dias ${ }^{17}$, a aparente gravidade dos munícipes de Praia Grande com EM merece investigação aprofundada.

Como ponto forte deste estudo citamos a potencial aplicabilidade dos resultados no planejamento de saúde do município de Praia Grande, visto que a pesquisa por meio do DATASUS retornou informações relevantes que podem contribuir para a busca ativa e acompanhamento desses 
munícipes. Como limitações, apontamos as restrições inerentes à mesma plataforma de busca, pois não é possível obter informações sobre as causas da internação. Além disso, não é possível saber se há readmissões hospitalares em meio às 57 internações reportadas, nem quantas vezes o mesmo indivíduo foi internado ao longo dos anos. Ainda assim, as informações podem ser valiosas para a elaboração de políticas públicas municipais e regionais voltadas às pessoas com EM.

\section{CONCLUSÕES}

A maioria dos munícipes de Praia Grande que foi internada por Esclerose Múltipla no período estudado era composta por adultos jovens do sexo masculino, com tempo médio ajustado de internação de 8,9 dias. Todas as internações ocorreram em municípios diferentes do de residência do usuário. O tempo de internação prolongado deve ser motivo para preocupações acerca do risco de complicações funcionais.

Sugerimos a elaboração de políticas públicas municipais e regionais para atender às demandas das pessoas com Esclerose Múltipla, de modo que o atendimento em todos os níveis de atenção possa ser dispensado o mais próximo possível da residência dos usuários.

Ressaltamos, também, a importância das informações fornecidas pelos estabelecimentos de saúde para 0 DATASUS, de modo a contribuir para o planejamento de ações voltadas às necessidades da população. Da mesma 
maneira, considerando a utilidade das informações disponibilizadas pelo DATASUS para a elaboração de políticas públicas e planejamento de cuidados, seria interessante que o sistema permitisse a inclusão da forma de EM entre os diagnósticos.

\section{REFERÊNCIAS}

1.Trapp BD, Peterson J, Ransohoff RM, Rudick R, Mörk S, Bö L. Axonal Transection in the Lesions of Multiple Sclerosis. New Eng J Med 1998;338:278-85. http://dx.doi.org/10.1056/nejm199801293380502 2. Correale J, Gaitan MI, Ysrraelit MC, Fiol MP. Progressive multiple sclerosis: from pathogenic mechanisms to treatment. Brain 2017;140:527-46. http://dx.doi.org/10.1093/brain/aww258

3.Lotti CBDC, Oliveira ASB, Bichuetti DB, Castro ID, Oliveira EML. Late onset multiple sclerosis: concerns in aging patients. Arq Neuropsiqu 2017;75:451-6. http://dx.doi.org/10.1590/0004-282x20170070

4.Oliveira EMLD, Souza NAD. Esclerose Múltipla. Rev Neurocienc 1998;6:114-8. http://dx.doi.org/10.34024/rnc.1998.v6.10324

5.Finkelsztejn A. Tratamento do surto de esclerose múltipla em hospital-dia: estudo de custo-minimização (Mestrado). Porto Alegre: Universidade Federal do Rio Grande do Sul; 2007. https://www.lume.ufrgs.br/bitstream/handle/10183/13414/00064268 $\underline{\text { 8.pdf }}$

6.BRASIL, Ministério da Saúde. (endereço na Internet) DATASUS Trajetória 1991-2002. Brasília: Ministério da Saúde; 2002 (acessado em: 17/10/21). Disponivel em: https://bvsms.saude.gov.br/bvs/publicacoes/trajetoria datasus.pdf

7.BRASIL, Ministério da Saúde. (endereço na Internet) DATASUS Departamento de Informática do SUS: Histórico (acessado em: 15/10/21). Disponível em: https://datasus.saude.gov.br/sobre-odatasus

8. Moreira MA, Felipe E, Mendes MF, Tilbery CP. Esclerose múltipla: estudo descritivo de suas formas clínicas em 302 casos. Arq Neuropsiqu 2000;58:460-6. http://dx.doi.org/10.1590/s0004282x2000000300010

9.Ribeiro TAGJ, Duarte AL, Silva DJD, Borges DJ, Costa VM, PapaisAlvarenga RM, et al. Prevalence of multiple sclerosis in Goiânia, Goiás, Brazil. Arq Neuropsiqu 2019;77:352-6. http://dx.doi.org/10.1590/0004-282x20190032

10.Luchetti S, Fransen NL, Van Eden CG, Ramaglia V, Mason M, Huitinga I. Progressive multiple sclerosis patients show substantial lesion activity that correlates with clinical disease severity and sex: a 
retrospective autopsy cohort analysis. Acta Neuropathol 2018;135:511-28. http://dx.doi.org/10.1007/s00401-018-1818-y

11.Voskuhl RR. The effect of sex on multiple sclerosis risk and disease progression. Mult Scler J 2020;26:554-60. http://dx.doi.org/10.1177/1352458519892491

12. Multiple Sclerosis International Federation - MSIF (endereço na internet). Atlas of MS 2013. Mapping multiple sclerosis around the world; 2013 (acessado em 15/10/21). Disponivel em: https://www.msif.org/wp-content/uploads/2014/09/Atlas-of-MS.pdf

13.Comissão de Incorporação de Tecnologias no SUS - CONITEC. (endereço na internet). Protocolo Clínico e Diretrizes Terapêuticas Esclerose Múltipla, 2019 (acessado em: 15/10/21). http://conitec.gov.br/images/Relatorios/2019/Relatorio_PCDT_EM_FI NAL.pdf

14.Ferreira MLB, Machado MÍM, Vilela ML, Guedes MJ, Ataide Jr L, Santos S, et al. Epidemiologia de 118 casos de esclerose múltipla com seguimento de 15 anos no centro de referência do Hospital da Restauração de Pernambuco. Arq Neuropsiqu 2004;62:1027-32. http://dx.doi.org/10.1590/s0004-282x2004000600018

15. Marques NI, Amancio AVP, Oikawa H, Leite MP, Peraro GB. Prevalência da esclerose múltipla na região Sul: um estudo epidemiológico. Apresentado no $1^{\circ}$ Congresso Interligas de Medicina UNINGÁ; 2020.

http://revista.uninga.br/index.php/uninga/article/view/3843

16. Cintra MMM, Mendonça AC, Rossi e Silva RC, Abate DT. Influência da fisioterapia na síndrome do imobilismo. Colloq Vitae 2014;5:9. https://journal.unoeste.br/index.php/cv/article/view/874

17.Patel S, SirDeshpande P, Desai R, Desai N, Mistry H, Patel N, et al. Thirty-day readmissions in multiple sclerosis: An age and genderbased US national retrospective analysis. Mult Scler Relat Disord 2019;31:10. http://dx.doi.org/10.1016/j.msard.2019.03.012 